\title{
Studying Materials for Applications in Next Generation Reactor Environments
}

\author{
J. A. Aguiar ${ }^{1,2}$, B. Hanken ${ }^{1}$, M. Asta ${ }^{1}$, M. Fluss ${ }^{2}$, L. Hsiung ${ }^{2}$, and N. D. Browning ${ }^{1,2,3}$ \\ ${ }^{1}$ Dept. Chemical Engineering \& Materials Science, University of California-Davis, Davis, CA 95616 \\ ${ }^{2}$ Lawrence Livermore National Laboratory, 7000 East Avenue, Livermore, CA 94550 \\ ${ }^{3}$ Dept. Molecular \& Cellular Biology, University of California-Davis, Davis, CA 95616
}

Since the inception of nuclear power, issues involving waste control and disposal have posed serious practical, financial, security, health and safety problems, due to the long lifetime and high radiotoxicity of spent nuclear fuel. To alleviate the growing demands for energy while taking into account environmental and potential security concerns, a series of next generation fusion and fast-spectrum fission reactors have been proposed. The next generation of redesigned fission reactors will transmute radiotoxic constituents while producing emission-free power under extreme operating conditions. To meet the strict criteria required for safe use of this fast reactor technology, several materials issues are being investigated, from the minor actinide containing oxide fuel sources to damage resistant oxide dispersed steels.

Consequently, there have been growing efforts to apply modern first-principles materials modeling tools as a framework for accelerated design and development of advanced new fuel and alloy-steel architectures for reactor technology. Following the materials modeling efforts, establishing a directly validated evolutionary model for these new reactor environments currently suffers from a lack of experimental validation. The absence of potential models that accurately address energetic contributions caused by swift ions further suspends the theoretical community. Direct validation of the purposed theoretical techniques, in their application to nuclear fuels and radiation resistant steels, would benefit from direct experimental measurements at the nanometer scale - in particular predictions related to the atomic and electronic structure of relevant point defects and complexes.

In order to address these deficiencies, we have commenced experimental and theoretical research to develop an empirical physical damage description for purposed nuclear fuel and steel elements. Samples studied thus far have included porous depleted urania, ceria, zirconia, yttria, thoria, and a variety solid solutions, including ceria-urania a surrogate for urania-plutonia. The experimental technique we use to study these materials is high angle annular dark field and bright field scanning transmission electron microscopy (STEM) paired with electronic energy loss spectroscopy (EELS). To increase spatial and energy resolution, the monochromated and Cs corrected FEI Titan at Lawrence Livermore National Laboratory and Cs corrected JEOL 2100 at UC Davis have been used.

Within the density functional theoretical framework, we employ a variety of calculations for studying the atomic structure associated with oxides. For the purposes of this presentation the latest hybrid and generalized gradient approximations with unique considerations are presented. The theoretical considerations include correlated f-states, magnetic ordering, spin-orbit coupling, and in view of the direct comparison to STEM/EELS the partial to full core-hole effect. To directly compare experiment and theory the core EELS oxygen $K$ - edge is chosen since the peak is most energetically sensitive to induced defects, vacancies, and complexes

The initial research into these materials complements an anticipated vision to characterize oxide fuels and alloys using the STEM/EELS experimental technique and theory. Primarily electronic 
structure calculations assist in unraveling the plausible local atomic contributions on the measured oxygen $K$-edge, as shown for example in Fig 1 , for a series of first-principles calculations varying the degree of correlation for primitive urania using the generalized gradient and alternate approaches. Initial experimental results further indicate a strong mixed spectral character, shown in Fig 2, in the near edge fine structure in a urania-ceria solid solution suggestive of multiple oxidation states. In the near future, the oxygen $K$-edge fine structure will be used to quantify radiation-induced segregation and meta-stable configurations in both fuel oxides and oxide-dispersed steels. This investigation into next generation nuclear energy related materials will be presented [1].

\section{References}

[1] Aspects of this research were supported by the US Department of Energy Nuclear Energy Research Initiative for Consortia (NERI-C) contract number DR-FG07-071D14893 and were performed under the auspices of the U.S. Department of Energy by Lawrence Livermore National Laboratory under Contract DE-AC52-07NA27344.

Comparing Experimental and Theoretical EELS Spectra

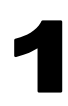

Urania Oxygen K-Edge Electron Near Fine Structure

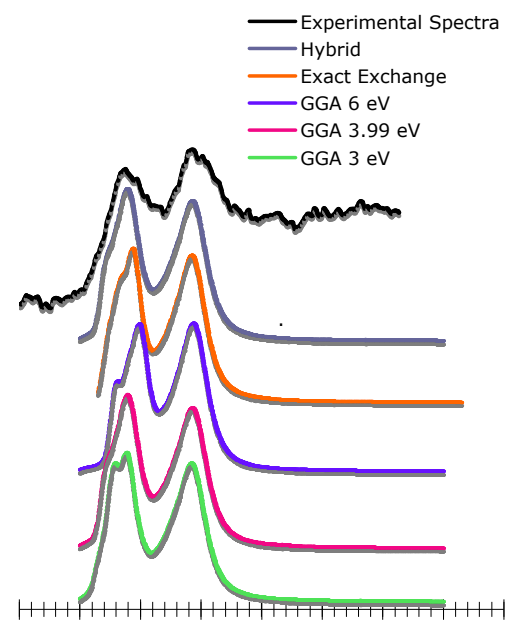

$\begin{array}{lllllllll}-5 & 0 & 5 & 10 & 15 & 20 & 30 & 35\end{array}$

Energy Beyond Edge Onset (eV)

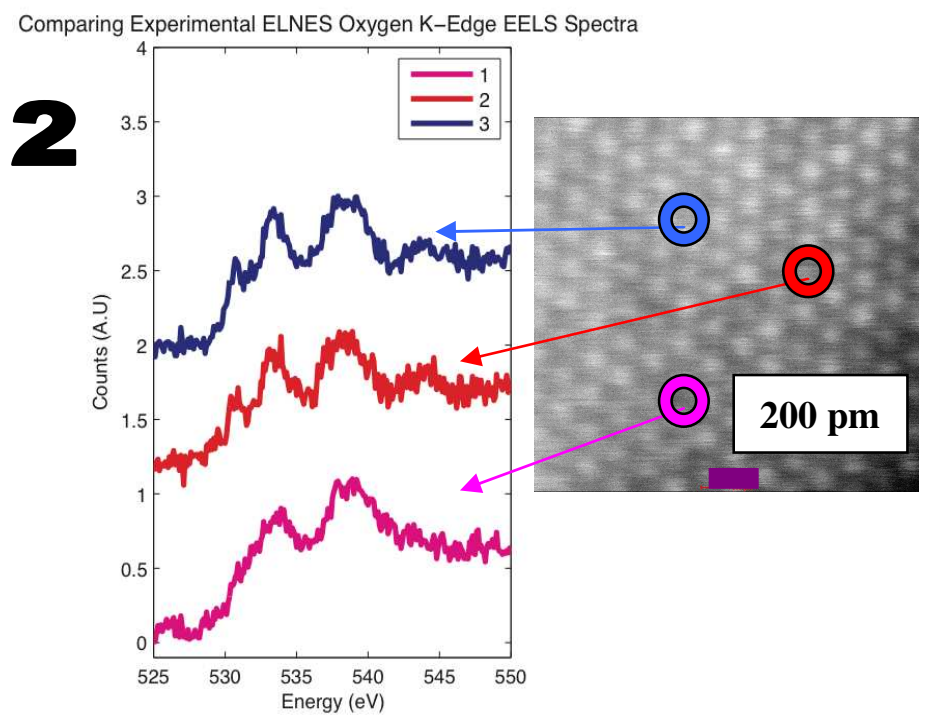

FIG.1. Experimental and

theoretically

resolved

oxygen $\mathrm{K}$ - near edge spectra for urania using various approximations to treat correlated fstates the linear augmented plane wave approach in Wien2k.

FIG.2. Simultaneously acquired atomic level STEM image and point resolved EELS spectra show mixed spectral character in the near oxygen $K$-edge in a uraniaceria system. 